\title{
CORRIGENDUM: ON CONVOLUTION OF BOAS TRANSFORM OF WAVELETS
}

\author{
NIKHIL KHANNA ${ }^{\dagger}$ AND LEENA KATHURIA
}

Date of Receiving : 28. 03. 2020

The paper [1] requires some clarifications. In Theorem 3.3 and Theorem 3.7, we assumed $G(x)=\int_{-1-x}^{1-x} \widehat{\psi^{(1)}}(\gamma) d \gamma$. This was incorrect. The correct value is given by $G(x)=\int_{-1}^{1}\left(1-\frac{1}{|\gamma|}\right) e^{-2 \pi i \gamma x} \widehat{\psi_{2}^{(1)}}(-\gamma) d \gamma$. For clarity we give the statements of these two theorems and also first few corrected lines of the proof of Theorem 3.3.

Theorem 3.3. Let $\psi_{1}, \psi_{2}$ be wavelets such that

(i) $\psi_{i}, \hat{\psi}_{i} \in L^{1}(\mathbb{R})$ for $i=1,2$,

(ii) $\hat{\psi}_{2}(0)=0$ and $\psi_{2}^{(1)} \in L^{1}(\mathbb{R})$, and

(iii) $\int_{\mathbb{R}} x^{q} G(x) d x=0$, for $0 \leq q \leq n$, where $G(x)=\int_{-1}^{1}\left(1-\frac{1}{|\gamma|}\right) e^{-2 \pi i \gamma x} \widehat{\psi_{2}^{(1)}}(-\gamma) d \gamma$. If $\psi_{1}$ and $\psi_{2}$ have $m$ and $n$ vanishing moments, respectively, then $\mathcal{B}\left\{\psi_{1} * \psi_{2}\right\}$ has $(m+n)$ vanishing moments provided that $x^{n} \psi_{2}(x) \in L^{2}(\mathbb{R})$.

Proof. We have

$$
\begin{aligned}
& \int_{\mathbb{R}} x^{q} \mathcal{B}\left\{\psi_{1} * \psi_{2}\right\}(x) d x= \sum_{p=0}^{q}{ }^{q} C_{p} \\
& \operatorname{Mom}_{p}\left(\psi_{1}\right)\left(\operatorname{Mom}_{q-p}\left(\mathcal{H} \psi_{2}\right)\right. \\
&\left.-\int_{\mathbb{R}} x^{q-p} \int_{\mathbb{R}} \mathcal{F}\left\{\mathcal{H} T_{-x} \psi_{2}\right\}(-\gamma) \hat{g}(\gamma) d \gamma d x\right) \\
&=\sum_{p=0}^{q}{ }^{q} C_{p} \operatorname{Mom}_{p}\left(\psi_{1}\right)\left(\operatorname{Mom}_{q-p}\left(\mathcal{H} \psi_{2}\right)\right. \\
&\left.+i \int_{\mathbb{R}} x^{q-p} \int_{-1}^{1}\left(1-\frac{1}{|\gamma|}\right) \mathcal{F}\left\{T_{-x} \psi_{2}^{(1)}\right\}(-\gamma) d \gamma d x\right) .
\end{aligned}
$$

2010 Mathematics Subject Classification. 42A38, 42C40, 44A15, 44A60.

Key words and phrases. Boas transform; wavelets; Hilbert transform; Fourier transform; vanishing moments.

Communicated by. Shiv K. Kaushik

${ }^{\dagger}$ Corresponding author. 